\title{
Incidentally Discovered HCC (iHCC) in Explant Liver-Histopathologic Features and Clinical Outcome
}

\author{
Renumathy Dhanasekaran ${ }^{1}$, Davinderbir S. Pannu ${ }^{1}$, Alpna Limaye ${ }^{2}$, Ivan Zendejas ${ }^{2}$, Roberto J. Firpi ${ }^{3}$, \\ Consuelo Soldevila-Pico ${ }^{3}$, Giuseppe Morelli ${ }^{3}$, Virginia C. Clark ${ }^{3}$, Amitabh Suman ${ }^{3}$, David R. Nelson ${ }^{3}$, \\ Roniel Cabrera $^{3^{*}}$
}

${ }^{1}$ Department of Medicine, University of Florida, Gainesville, USA; ${ }^{2}$ Department of Surgery, University of Florida, Gainesville, USA; ${ }^{3}$ Division of Gastroenterology, Hepatology and Nutrition, University of Florida, Gainesville, USA.

Email: *rcabrera@ufl.edu

Received January $10^{\text {th }}, 2013$; revised February $8^{\text {th }}, 2013$; accepted February $16^{\text {th }}, 2013$

\begin{abstract}
Incidentally discovered hepatocellular carcinomas (iHCC) are tumors which are discovered on the explanted liver which were not present on imaging prior to transplant. The natural history, histopathologic characteristics and prognosis of iHCC are not clearly defined. Methods: A retrospective analysis was performed to compare the characteristics of iHCC and established HCC within Milan criteria (eHCC) in patients who underwent liver transplantation at our center between 2000 and 2010. Results: During the study period a total of 975 adult patients were transplanted in our center; 124 (12.7\%) patients had eHCC and 26 (2.6\%) patients had iHCC. A larger number of patients with iHCC (73.1\%) had ascites when compared to eHCC $(41.3 \%)(p=0.035)$. Patients with iHCC had a higher mean bilirubin $(\mathrm{p}<0.001)$ and mean INR $(p=0.05)$ than patients with eHCC. Around $70 \%$ of patients with iHCC had a Model for End Stage Liver Disease (MELD) score greater than 15 at the time of listing while only 25\% of patients with eHCC had a MELD greater than 15 at listing $(\mathrm{p}<0.001)$. The mean alphafetoprotein (AFP) in patients with iHCC was significantly lower $(11.6 \pm$ $16.5)$ than the patients with eHCC $(564.9 \pm 2180 ; p=0.024)$. In patients with iHCC, $30.8 \%$ had a multiple tumors and 23\% had bilobar involvement. The average number of tumors was 1.6 (1 - 5 What this Range?) and the cumulative tumor size was $2.1 \mathrm{~cm}$ (SD 1.4). The cumulative tumor size in iHCC was significantly smaller than in eHCC (mean 3.9 $\mathrm{cm})(\mathrm{p}=0.035)$. American Joint Committee on Cancer (AJCC) T1 tumor stage was found in $58 \%$ of patients with iHCC and $48.4 \%$ of patients with eHCC ( $\mathrm{p}=0.829)$. The median survival was 9.47 years for iHCC (95\% CI 7.0 - 11.9) and 8.7 years $(95 \%$ CI 6.1 - 11.4) for eHCC ( $=0.328)$. While none of the patients with iHCC had recurrence of HCC, the incidence of recurrence in patients with eHCC was $6.4 \%$. Conclusion: iHCC occurred in patients with more advanced liver disease than eHCC. AFP was usually not elevated in patients with iHCC. The cumulative tumor size of iHCC was smaller than eHCC but around a third of iHCC were multifocal, supporting the theory of multicentric hepatic carcinogenesis. Survival of patients with iHCC was similar to patients with eHCC and recurrence was not noted in patients with iHCC.
\end{abstract}

Keywords: Incidental; HCC; Recurrence; Survival

\section{Introduction}

Hepatocellular carcinoma (HCC) is a cancer of global importance with an incidence that is on the rise [1]. In the United States, the incidence has tripled over the last three decades with over 20,000 cases estimated to be diagnosed in 2011 [2]. The best curative option for HCC is early diagnosis, followed by transplantation when the tumor burden is still within the Milan criteria (defined as having one lesion $<5 \mathrm{~cm}$ or up to three lesions $<3 \mathrm{~cm}$ in diameter each) [3,4]. But sometimes HCC is not diagnosed in the pretransplant evaluation and it is inciden-

${ }^{*}$ Corresponding author. tally found in the explant liver. In this study we are exploring these patients who are incidentally diagnosed to have HCC after liver transplantation.

Ultrasound has been recommended for population based screening as it is inexpensive and easily available. Despite strong recommendations, there are a considerable number of cirrhotics who are not being screened for HCC. In a recent population-based cohort identified from the linked Surveillance, Epidemiology, and End Results registry (SEER)-Medicare databases, less than 20\% of patients who developed HCC had received regular surveillance [5]. Though ultrasound is the modality used for screening, its sensitivity in detecting HCC is intermediate 
and has been reported to vary between $30 \%-70 \%[6,7]$. For patients who have undergone CT (computed tomography) or MRI, there has been concern regarding sensitivity in detecting small tumors [8]. So a combination of lack of screening and low sensitivity of radiologic tests can lead to the diagnosis of HCC being missed in the pretransplant period.

Incidentally discovered HCCs (iHCC) are tumors newly discovered on explant liver in patients who were transplanted for other benign indications. Lack of screening is probably not a contributor in this population as patients on the transplant list do have close follow up, and almost all of them have pretransplant liver imaging. The difficulty in diagnosing small HCC, as mentioned above, partly explains the occurrence of iHCC. Is the patient with iHCC different from patients with established HCC (eHCC)? This is a question of clinical relevance to patients who receive this diagnosis post-transplant. There are a few studies which have reported clinical outcomes of patients with iHCC but there is wide discrepancy in data [9-13]. In this study, we have tried to clarify some important aspects related to iHCC ranging from clinical features, to tumor characteristics and treatment outcome.

\section{Methods}

\subsection{Patient Population}

Nine hundred and seventy five adult liver transplants were performed at our center from 2000 to 2010. Our study population was limited to the 150 consecutive adult liver transplants (LT) performed for HCC during this time period. All other indications for LT were excluded from our study population. The study was approved by the Institutional Review Board at the University of Florida, Gainesville, FL.

\subsection{Data Collection}

The following data were collected for all patients: 1$)$ demographics-age, sex, ethnicity; 2) tumor-related datasize and number of lesions in pretransplant imaging, occurrence of recurrence after LT; 3) pathologic features on explants - grade of tumor, degree of differentiation, and microvascular invasion; 4) laboratory parameters including liver function tests, creatinine, and serum [alpha]-fetoprotein (AFP) before transplant; 5) transplant datadate of listing and date of transplant; 6) post transplant outcomes measures-recurrence and survival data.

\subsection{Pre Transplant Diagnosis}

HCC was diagnosed according to the non-invasive radiologic criteria based on the AASLD guidelines [4]. All patients had a CT or MRI within 6 months of transplant, including those with iHCC. Only patients with tumor bur- den within Milan criteria were considered eligible for transplantation.

\subsection{Explant Histopathology}

For patients with iHCC, date of transplant was used as date of diagnosis. Pathologic examination was carried out on all explanted livers. They were examined for tumor size and number, tumor grade, histologic differentiation, and presence of vascular invasion. The American Joint committee on Cancer (AJCC)/International Union Against Cancer (UICC) staging system (6th edition) was used for staging the tumor in the explant [14]. For multifocal tumors, the cumulative tumor size was calculated by adding the longest diameter of each individual tumor.

\subsection{Immunosuppression Regimens}

Our institutional standard immunosuppression regimen consists of tacrolimus and prednisone. Immunosuppression was typically tapered to monotherapy (tacrolimus or cyclosporine) within four to six months of transplantation. Si- rolimus was also used when chronic renal insufficiency occurred during prolonged follow-up after LT. No switch in immunosuppression was made based on diagnosis of HCC.

\subsection{Follow Up}

After transplantation, all patients were followed up closely in clinic with blood draw for chemistries and AFP. They were screened for recurrence at 6 month intervals with ultrasound. If there was clinical suspicion for distant metastases then appropriate scans were ordered.

\subsection{Study Objectives}

The primary objective of the study was to compare the clinical presentation, histologic features and post-transplant outcome of patients with iHCC and eHCC.

\subsection{Statistical Analysis}

The chi square test was used to compare categorical variables and student $t$ tests were used to compare continuous variables. Survival time was defined as the time from the time of transplant to the date of death or the date of last follow-up. Survival analysis was carried out using Kaplan-Meier Estimator method. Survival estimates were compared with log rank test in the Kaplan-Meier Estimator method. The SPSS Graduate version 16.0 (SPSS Inc., Chicago, IL) was used for all statistical analysis.

\section{Results}

During the study period of 2000-2010, a total of 975 patients were transplanted in our center and 150 (15.4\%) of 
them had HCC. The incidence of iHCC in the study group was 2.6\% $(\mathrm{n}=26)$. Table 1 compares the demographic and clinical features between patients with iHCC and eHCC. Overall a majority of patients in both groups were Caucasian $(80.0 \%)$ males $(81.3 \%)$ and their distri-

Table 1. Comparison of demographic and clinical features of incidentally discovered Hepatocellular Carcinoma (iHCC) and Previously Known HCC (eHCC).

\begin{tabular}{|c|c|c|c|c|}
\hline Variable & Category & $\begin{array}{c}\text { iHCC } \\
(n=26)\end{array}$ & $\begin{array}{c}\text { eHCC } \\
(n=124)\end{array}$ & $P$ value \\
\hline \multirow{2}{*}{ Gender } & Male & 18 & 104 & \multirow{2}{*}{0.098} \\
\hline & Female & 8 & 20 & \\
\hline \multirow{4}{*}{ Race } & Caucasian & 22 & 98 & \multirow{4}{*}{0.171} \\
\hline & $\begin{array}{l}\text { African } \\
\text { American }\end{array}$ & 3 & 5 & \\
\hline & Hispanic & 0 & 7 & \\
\hline & Others & 1 & 14 & \\
\hline \multirow{2}{*}{ Ascites } & Yes & 19 & 52 & \multirow{2}{*}{0.035} \\
\hline & No & 7 & 68 & \\
\hline \multirow{2}{*}{ Encephalopathy } & Yes & 11 & 30 & \multirow{2}{*}{0.212} \\
\hline & No & 14 & 91 & \\
\hline \multirow{2}{*}{ Varices } & Yes & 10 & 51 & \multirow{2}{*}{0.858} \\
\hline & No & 16 & 70 & \\
\hline \multirow{2}{*}{ Hepatitis C } & Yes & 15 & 92 & \multirow{2}{*}{0.100} \\
\hline & No & 11 & 32 & \\
\hline \multirow[b]{2}{*}{ Number of tumors } & Single & 18 & 95 & \multirow[b]{2}{*}{0.517} \\
\hline & Multiple & 8 & 27 & \\
\hline \multirow{2}{*}{ Lobar involvement } & Unilobar & 20 & 106 & \multirow{2}{*}{0.346} \\
\hline & Bilobar & 6 & 16 & \\
\hline \multirow{2}{*}{$\begin{array}{l}\text { Microvascular } \\
\text { invasion }\end{array}$} & No & 21 & 95 & \multirow{2}{*}{0.222} \\
\hline & Yes & 5 & 17 & \\
\hline \multirow{2}{*}{ T stage } & $\mathrm{T} 1$ & 15 & 61 & \multirow{2}{*}{0.829} \\
\hline & T2-T3 & 11 & 51 & \\
\hline $\begin{array}{l}\text { Average number of } \\
\text { tumors }\end{array}$ & Mean (SD) & $1.6(1.1)$ & $1.4(0.9)$ & 0.104 \\
\hline $\begin{array}{c}\text { Cumulative tumor } \\
\text { size }\end{array}$ & Mean (SD) & $2.1(1.4)$ & $3.9(2.0)$ & 0.031 \\
\hline Age & Mean (SD) & $55.7(10.1)$ & 55.1 (7.9) & 0.891 \\
\hline Albumin & Mean (SD) & $2.8(0.4)$ & $3.3(0.6)$ & 0.090 \\
\hline Bilirubin & Mean (SD) & 5.7 (6.7) & $2.4(2.1)$ & $<0.001$ \\
\hline Creatinine & Mean (SD) & $1.2(0.4)$ & $1.0(1.1)$ & 0.586 \\
\hline AFP & Mean (SD) & 11.6 (16.5) & 564.9 (2180) & 0.024 \\
\hline INR & Mean (SD) & $1.6(0.3)$ & $1.3(0.2)$ & 0.050 \\
\hline MELD & Mean (SD) & $18.3(5.5)$ & $12.9(4.9)$ & 0.476 \\
\hline
\end{tabular}

bution between the two groups was similar. The mean age of patients in the study group was $55.2 \pm 8.3$ yrs and there was no difference in age between the two groups. The etiology of cirrhosis in patients with iHCC were hepatitis $C(n=15)$, alcoholic liver disease $(n=6)$, hepatitis B ( $n=2)$, non-alcoholic steatohepatitis $(n=2)$ and others $(\mathrm{n}=1)$.This distribution was also similar to patients with eHCC. Hepatitis $\mathrm{C}$ was the most common underlying etiologic category in both groups (58\% in iHCC and 73\% in eHCC). The mean time from diagnosis of eHCC to transplant was 2.9 months (4 days - 44 months).

The presence of ascites, encephalopathy and esophageal varices were recorded in all patients. Larger number of patients with iHCC (73.1\%) had ascites when compared to eHCC $(41.3 \%)(p=0.035)$. Hepatic encephalopathy was present in $42.3 \%$ and varices were present in $38.4 \%$ of patient with iHCC, but this was similar to eHCC. Liver synthetic function appeared to be worse in patients with iHCC. Patients with iHCC had a higher mean bilirubin $(p<0.001)$ and mean INR $(p=0.05)$ than patients with eHCC. Around $70 \%$ of patients with iHCC had a Model for End Stage Liver Disease (MELD) score greater than 15 at the time of listing while only $25 \%$ of patients with eHCC had a MELD greater than 15 (p < 0.001).

The mean AFP in patients with iHCC was 11.6 (SD 16.5), while it was significantly higher in patients with eHCC 564.9 (SD 2180) ( $p=0.024$ ). In patients with iHCC $30.8 \%$ had multiple tumors and $23 \%$ had bilobar involvement. The cumulative tumor size in iHCC was 2.1 $\mathrm{cm}$ and this was significantly less than eHCC (mean 3.9 $\mathrm{cm})(\mathrm{p}=0.035)$. When individual tumor nodules were considered, $80.8 \%(21 / 26)$ of patients had tumors less than $2 \mathrm{~cm}$ and $42.3 \%(11 / 26)$ had tumors less than $1 \mathrm{~cm}$ in diameter. The incidence of microvascular invasion was not different between iHCC (19.2\%) and eHCC (13.7\%) $(p=0.222)$. AJCC T1tumor stage was found in $58 \%$ of patients with iHCC and $48.4 \%$ of patients with eHCC (p $=0.829$ ).

Patients were followed for a mean duration of $4.27 \mathrm{yrs}$ (range 0.1 - $12.5 \mathrm{yrs}$ ). The median survival of patients with iHCC was 9.47 years (95\% CI, 7.0 - 11.9) and it was 8.7 years $(95 \%$ CI 6.1 - 11.4) in patients with eHCC (p = 0.328 ). The one and five year survival in patients with iHCC was $81 \%$ and $77 \%$, respectively, and this was not significantly different from patients with eHCC (85\% and $63 \%)$. The incidence of recurrence in patients with eHCC was $6.4 \%$ and none of the patients with iHCC had recurrence of $\mathrm{HCC}$.

\section{Discussion}

In our study we found a low incidence of only $2.6 \%$ for iHCC. We also found that patients with iHCC had excel- 
lent post-transplant survival. The post-transplant survival in patients with iHCCwas similar to patients with eHCC. Further, there was no recurrence in the patients with iHCC.

The low rate of iHCC in our study is very much in line with previous reports. The rate of iHCC has varied widely in different case series, with older studies reporting higher incidence. One of the highest incidences was described in a study from 1996, where they reported an incidence of iHCC of $17.5 \%$ among 80 examined explants [15]. But more recent studies describe an incidence between 1.0\% $6.7 \%$ with a median of $2.6 \%$ [9]. Other studies have reported similar results $[9,10]$ but there are a few studies which have shown that patients with iHCC actually do better than patients with eHCC [16]. This is probably attributed to the reported lower incidence of recurrence in the iHCC group $[9,10]$.

We also found that the patients with iHCC had more advanced liver disease than patients with eHCC. The incidence of ascites was higher in iHCC, and patients also had a higher MELD score indicating worse synthetic function. The OPTN (Organ Procurement and Transplantation Network) and UNOS (United Network for Organ Sharing) use the MELD score for prioritizing organ sharing, as it is an indicator of short-term mortality. However, for patients with HCC, falling off the wait list due to the risk of tumor progressing beyond the Milan criteria influences more the short-term mortality than the liver synthetic function. [17]. So patients with pretransplant HCC get an exception pathway and are allocated a MELD score of 22 even if their original score is lower [18]. In our study, the median time from diagnosis of HCC to transplant was 2.9 months in patients with eHCC. So it is likely that most of these patients got this exception pathway to transplant, which explains why their MELD scores are lower than that of iHCC patients. In addition, HCC is known to occur in more advanced cirrhosis as the progressive scarring and inflammation lead to a tumorigenic milieu $[19,20]$. Thus, patients with iHCC possibly were in early stages of carcinogenesis during their wait on the transplant list.

The cumulative tumor size of the iHCC in our study was statistically smaller than that of eHCC. This finding is probably due to the poor sensitivity of radiologic studies to detect smaller tumors. Once a lesion is detected on ultrasound, cross sectional imaging with multiphase CT is the most commonly used modality to confirm the diagnosis of HCC. Radiologic studies have reported varying sensitivities for the detection of lesions even with multiphase CT, with studies reporting lower sensitivities for smaller lesions [1]. In one study the detection rate of multidetector CT (MDCT) dropped from 98\% (larger than $20 \mathrm{~mm}$ ) to $78 \%$ for lesions smaller than $10 \mathrm{~mm}$ [21]. MRI has been another promising modality to diagnose
HCC but it is not easily available and it is more costly. Similarly, MRI also has poor sensitivity to detect small lesions that can be as low as 33\% [22]. The concern for the poor sensitivity of radiologic studies for small lesions is widely acknowledged. A recent review reported overall sensitivities for lesions less than $2 \mathrm{~cm}$ of various modalities to be $21 \%$ for US, $40 \%$ for enhanced CT and $47 \%$ for MRI [23]. The majority of the patients in the iHCC subgroup (more than 80\%) had nodules less than $2 \mathrm{~cm}$ which probably explains why these lesions were missed on pretransplant cross sectional imaging.

There has long been a debate whether multiple HCCs in cirrhotics are predominantly due to multifocal carcinogenesis or if they originate from a single tumor and spread intrahepatically through metastasis. For clonality analysis, previous studies have used DNA fingerprinting with loss of heterozygosity $(\mathrm{LOH})$ assay, comparative genomic hybridization (CGH), and hepatitis B virus (HBV) integration pattern [24-27]. But discrimination between these two types of carcinogenesis is usually difficult. In our study, almost a third of iHCC were multifocal and $23 \%$ had bilobar involvement. The presence of small multifocal tumors on the explant suggests these are possibly of multicentric origin and not from intrahepatic metastases.

Some of the limitations to this study include that it is retrospective and it is a single center study. The number of patients with iHCC is small but it still is larger than most case series in the literature. We do have a control group of patients with eHCC which helps contrast the two groups. And we are able to compare clinicopathological data with clinical outcome while many studies report one or the other. Overall, our study does bring up some interesting observations which throw further light on HCC tumorigenesis and highlight the limitations of radiologic diagnosis.

\section{Conclusion}

The occurrence of finding an iHCC in patients undergoing liver transplantation for decompensated liver disease is very low. When found on explants these HCCs are usually small in size but can be multifocal coinciding with the more advanced liver disease present in these patients and the theory of multicentric hepatic carcinogenesis. Importantly, the patients who are found to have incidental HCCs after a liver transplant have an excellent post-liver transplant survival,similar to the patients with established HCC within Milan who receive a liver transplant. Further, findings an iHCC on a liver explant does not appear to lead to recurrence after liver transplantation. This information will be valuable to reassure and counsel patients who receive this diagnosis as an intimidating surprise in the post-transplant period. 


\section{REFERENCES}

[1] R. Dhanasekaran and A. Limaye, "Hepatocellular Carcinoma: Current Trends in Worldwide Epidemiology, Risk Factors, Diagnosis, and Therapeutics," Hepatic Medicine: Evidence and Research, Vol. 4, 2012, pp. 19-37.

[2] Surveillance Research Program, National Cancer Institute, "Fast Stats: An Interactive Tool for Access to SEER Cancer Statistics,” 2011. http://seer.cancer.gov/faststats

[3] V. Mazzaferro, et al., "Liver Transplantation for the Treatment of Small Hepatocellular Carcinomas in Patients with Cirrhosis,” The New England Journal of Medicine, Vol. 334, No. 11, 1996, pp. 693-699. doi:10.1056/NEJM199603143341104

[4] J. Bruix and M. Sherman, "Management of Hepatocellular Carcinoma: An Update,” Hepatology, Vol. 53, No. 3, 2011, pp. 1020-1022. doi:10.1002/hep.24199

[5] J. A. Davila, et al., "Use of Surveillance for Hepatocellular Carcinoma among Patients with Cirrhosis in the United States,” Hepatology, Vol. 52, No. 1, 2010, pp. 132-141. doi:10.1002/hep.23615

[6] E. K. Outwater, "Imaging of the Liver for Hepatocellular Cancer,” Cancer Control, Vol. 17, No. 2, 2010, pp. 72-82.

[7] A. Colli, et al., "Accuracy of Ultrasonography, Spiral CT, Magnetic Resonance, and Alpha-Fetoprotein in Diagnosing Hepatocellular Carcinoma: A Systematic Review," American Journal of Gastroenterology, Vol. 101, No. 3, 2006, pp. 513-523. doi:10.1111/j.1572-0241.2006.00467.x

[8] M. J. Kim, "Current Limitations and Potential Breakthroughs for the Early Diagnosis of Hepatocellular Carcinoma," Gut Liver, Vol. 5, No. 1, 2011, pp. 15-21. doi:10.5009/gnl.2011.5.1.15

[9] G. C. Sotiropoulos, et al., "Liver Transplantation and Incidentally Found Hepatocellular Carcinoma in Liver Explants: Need for a New Definition?” Transplantation, Vol. 81, No. 4, 2006, pp. 531-535. doi:10.1097/01.tp.0000198739.42548.3e

[10] Y. Kishi, et al., "Impact of Incidentally Found Hepatocellular Carcinoma on the Outcome of Living Donor Liver Transplantation,” Transplant International, Vol. 19, No. 9, 2006, pp. 720-725. doi:10.1111/j.1432-2277.2006.00338.x

[11] R. Raphe, et al., "Histopathologic Characteristics of Incidental Hepatocellular Carcinoma after Liver Transplantation,” Transplantation Proceedings, Vol. 42, No. 2, 2010, pp. 505-506. doi:10.1016/j.transproceed.2010.01.034

[12] S. H. Choi, et al., "Clinicopathological Features of Incidental Hepatocellular Carcinoma in Liver Transplantation,” Transplantation Proceedings, Vol. 36, No. 8, 2004, pp. 2293-2294. doi:10.1016/j.transproceed.2004.08.076

[13] A. Caroli-Bottino, et al., "Hepatocellular Carcinoma: Incidental Finding in Cirrhotic Explanted Livers,” Transplantation Proceedings, Vol. 37, No. 6, 2005, pp. 27912792. doi:10.1016/j.transproceed.2005.07.014

[14] F. L. Greene, D. L. Page, I. D. Fleming, A. Fritz, C. M. Balch, D. G. Haller, et al., "AJCC Cancer Staging Manual,” 6th Edition, Springer, Chicago, 2002.

[15] F. Mion, et al., "Adult Cirrhotic Liver Explants: Precancerous Lesions and Undetected Small Hepatocellular Car- cinomas," Gastroenterology, Vol. 111, No. 6, 1996, pp. 1587-1592. doi:10.1016/S0016-5085(96)70021-5

[16] J. A. Fernandez, et al., "Can We Expand the Indications for Liver Transplantation among Hepatocellular Carcinoma Patients with Increased Tumor Size?” Transplant Proceedings, Vol. 35, No. 5, 2003, pp. 1818-1820. doi:10.1016/S0041-1345(03)00723-1

[17] R. B. Freeman Jr., et al., "Model for End-Stage Liver Disease (MELD) Exception Guidelines: Results and Recommendations from the MELD Exception Study Group and Conference (MESSAGE) for the Approval of Patients Who Need Liver Transplantation with Diseases Not Considered by the Standard MELD Formula," Liver Transplantation, Vol. 12, No. S3, 2006, pp. S128-S136. doi:10.1002/lt.20979

[18] E. A. Pomfret, et al., "Report of a National Conference on Liver Allocation in Patients with Hepatocellular Carcinoma in the United States," Liver Transplantation, Vol. 16, No. 3, 2010, pp. 262-278. doi:10.1002/lt.21999

[19] A. J. Sanyal, S. K. Yoon and R. Lencioni, “The Etiology of Hepatocellular Carcinoma and Consequences for Treatment,” The Oncologist, Vol. 15, No. S4, 2010, pp. 14-22. doi:10.1634/theoncologist.2010-S4-14

[20] H. B. El-Serag, "Hepatocellular Carcinoma," New England Journal of Medicine, Vol. 365, No. 12, 2011, pp. 1118-1127. doi:10.1056/NEJMra1001683

[21] A. Luca, et al., "Multidetector-Row Computed Tomography (MDCT) for the Diagnosis of Hepatocellular Carcinoma in Cirrhotic Candidates for Liver Transplantation: Prevalence of Radiological Vascular Patterns and Histological Correlation with Liver Explants," European Radiology, Vol. 20, No. 4, 2010, pp. 898-907. doi:10.1007/s00330-009-1622-0

[22] G. A. Krinsky, et al., "Transplantation for Hepatocellular Carcinoma and Cirrhosis: Sensitivity of Magnetic Resonance Imaging,” Liver Transplantation, Vol. 8, No. 12, 2002, pp. 1156-1164. doi:10.1053/jlts.2002.35670

[23] N. C. Yu, et al., "CT and MRI Improve Detection of Hepatocellular Carcinoma, Compared with Ultrasound Alone, in Patients with Cirrhosis,” Clinical Gastroenterology and Hepatology, Vol. 9, No. 2, 2011, pp. 161-167. doi:10.1016/j.cgh.2010.09.017

[24] K. Takahashi, et al., "Frequent Loss of Heterozygosity on Chromosome 22 in Hepatocellular Carcinoma," Hepatology, Vol. 17, No. 5, 1993, pp. 794-799. doi:10.1002/hep.1840170508

[25] I. O. Ng, et al., "Determination of the Molecular Relationship between Multiple Tumour Nodules in Hepatocellular Carcinoma Differentiates Multicentric Origin from Intrahepatic Metastasis,” Journal of Pathology, Vol. 199, No. 3, 2003, pp. 345-353. doi:10.1002/path.1287

[26] M. Esumi, et al., "Clonal Origin of Human Hepatoma Determined by Integration of Hepatitis B Virus DNA," Cancer Research, Vol. 46, No. 11, 1986, pp. 5767-5771.

[27] O. Morimoto, et al., "Diagnosis of Intrahepatic Metastasis and Multicentric Carcinogenesis by Microsatellite Loss of Heterozygosity in Patients with Multiple and Recurrent Hepatocellular Carcinomas,” Journal of Hepatology, Vol. 39, No. 2, 2003, pp. 215-221. doi:10.1016/S0168-8278(03)00233-2 